I.

\title{
Marginalien I.
}

Daf3 Hor az a.p. 195 ff. speciell auf den sophokleischen Chor gehe, ist schon von anderen bemerkt worden; es fragt sich num, ob ihm bei seinen specielleren Angaben bestimmte Chorlieder des griechischen Dichters vorschwebten, oder nur eine allgemeine Empfindung von der Ausgestaltung, die er dem Chor gegeben hatte. Für die erstere Auffassung läfit sich folgendes $\mathrm{m}$. W. noch nicht wahrgenommene Moment geltend machen. In den Versen ille dapes laudet mensae brevis, ille salubrem Justitiam legesque et apertis otia portis sind die drei Begriffe justitia, leges, otia durch das gemeinsame ille zusammengefafat; nun ist es gewif kein Zufall, dafs sie auf grie-

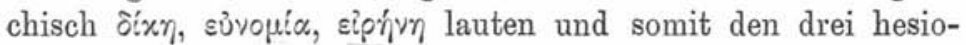
dischen Horen entsprechen. Der Römer konnte das aus der horazischen Uebersetzung nicht herausfinden; es ist daher wahrscheinlich, daf Horaz einen bestimmten, uns nicht erhaltenen sophokleischen Chorgesang im Auge hatte, der dem Lob der drei Horen galt.

$$
\text { *** }
$$

Servatus Lupus schreibt an Eginhart, nachdem er dessen Biographie Karls d. Gr. gelesen hatte, u. a. folgendes über den Stil der Schrift: ibi elegantiam sensuum, ibi raritatem conjunctionum, quam in auctoribus notaveram, .... amplexus sum. Die herausgehobenen Worte sind Norden (ant. Kunstprosa 707) unverständlich geblieben; mir scheint es jedoch, dafs eine im weiteren Verlaufe (S. 716) von ihm citirte 
Stelle aus Joannes Saresberiensis poetas aut oratores proponebat et eorum jubebat vestigia imitari ostendens juncturas dictionum et elegantes sermonum clausulas die Erklärung gibt: es ist die horatianische callida junctura, von deren Fortwirken im Mittelalter man sich auf diese Weise überzeugt. In ähnlichem Sinne sagte der ältere Goncourt einmal von einem Schriftsteller, er habe l'épithète rare.

Des Meeres und der Liebe Wellen. Sie sind in Horazens lieblicher Pyrrhaode (I 5) so reizend parallelisirt, daf man gewißs eine ganz kleine Aenderung nicht scheuen wird, um dem Gedicht zu seiner poetischen Vollendung zu verhelfen. Die erste Strophe ist ganz, von der Wirklichkeit nämlich der Pyrrha - ausgefüllt; mit der zweiten begeben wir uns allmählich auf den Boden der Allegorie, worauf in der dritten die Rückkehr zur Wirklichkeit erfolgt. Gegen das Ende hin spielt die Allegorie wieder neckisch drein;, wobei der Uebergang dieselbe kunstvoll abgetönte Allmählichkeit aufweist. Nun verlangen wir zum Abschluk einen Begriff, der beiderlei Strahlen in sich vereinigt; wer aufmerksam die letzten Verse liest

me tabula sacer

votiva paries indicat uvida

suspendisse potenti

vestimenta maris -

wird nicht zweifeln, daß der Dichter eben diesem Begriff zusteuerte: ist es doch dieselbe Göttin, der beide Elemente, das Meer und die Liebe, untertan sind - die Ven us marina, deren der Dichter, puellis nuper idoneus, III 22 bei weit weniger passender Gelegenheit gedenkt. Hier, wo vom Schiffbruch der Liebe die Rede ist, kann der Dichter vollends nur eins gewollt haben:

... potenti

vestimenta maris dea e.

* * *

Des Meeres und der Liebe Wellen haben wir auch bei Lucian, $\alpha \alpha \tau \alpha \pi \lambda .6$; doch ist auch hier eine Aenderung des

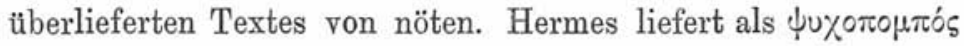


seine Todten gruppenweise der Klotho ab, nach den Todesarten geordnet; es kommen die Verurteilten dran, dann fordert Klotho die von Räubern erschlagenen. Hermes antwortet:

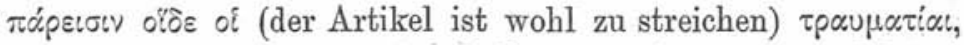

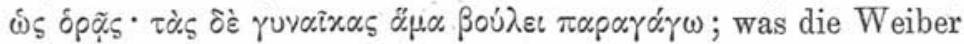
hier sollen, begreift man schwer: ihre Erwähnung verstößt gegen das Anordnungsprincip. Nun lesen wir aber weiter die

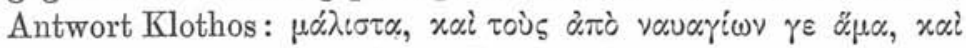

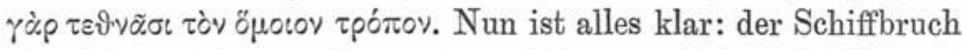
der Liebe wird auch hier, wie bei Horaz, mit dem Schiffbruch im Meere parallelisirt. Es ist daher in der Frage des Hermes

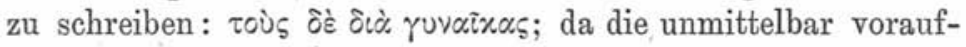

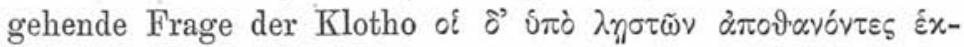
น

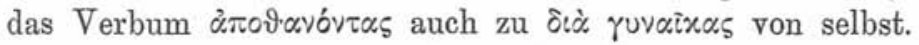

Dafs der vergilianische $\mathrm{Cul}$ ex einem griechischen Original nachgebildet sei, hat Maass (Orpheus $237 \mathrm{ff}$.) neuerdings mit guten Gründen zu erweisen gesucht; einen m. E. entscheidenden, den er nicht beachtet zu haben scheint, will ich hier nachtragen. Der todten Mücke gehn drei Heroinen, vorab Alcestis, entgegen (261 f.); warum gerade Heroinen? Bei einer Vibia begreift sich das - die Frau soll von Frauen geleitet werden; aber d e m parvus culex würde man eher Heroen wie Antilochus, Menoeceus, Protesilaus als Hadesführer wünschen. Gerade die von Maass S. 241 angeführten Belege lassen den Geschlechtswechsel unerklärt erscheinen; auch dem Leosthenes (S. 240) sollen nicht Heroinen, sondern Heroen entgegenschreiten. Mit der Annahme nun einer griechischen Vorlage ist alles erklärt: der hellenistische Dichter lief seine hingebende $\varepsilon \hat{\mu} \pi i i_{\varsigma}$ ganz naturgemäfs von Alkestis, Penelope und Eurydike geleiten.

$$
\text { * * * }
$$

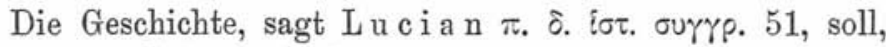
einem reinen Spiegel gleich, die Ereignisse zurückstrahlen: oủ

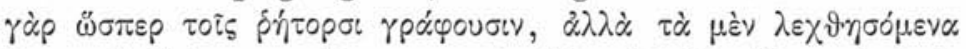

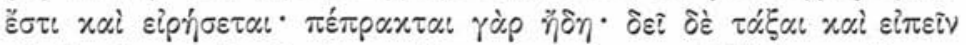

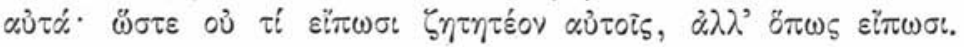




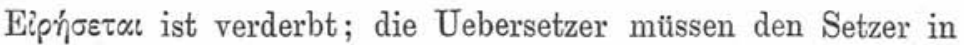
Mitleidenschaft ziehn, um irgend einen Sinn herauszubekommen (Hemsterhuys: dicenda praesto sunt et dicentur omnino; Wieland: sie sind schon da und müssen u. s. w.) oder nehmen ihre Zuflucht zu unerlaubten Interpolationen (Sommerbrodt: der Stoff ist vorhanden und soll [wie er ist, d. i. der Wahrheit gemäf]

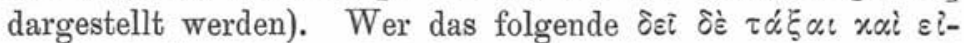

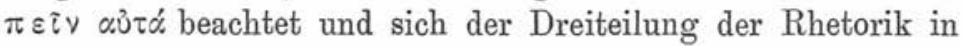

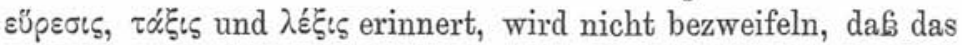

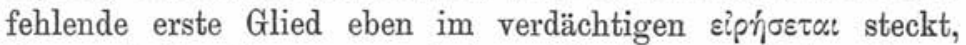

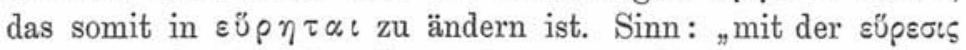
braucht sich der Historiker, darin vom Redner verschieden, nicht zu befassen: sein Stoff ist bereits da, er ist 'gefunden' eben dadurch, daf er geschehn ist; was ihm obliegt, ist einzig und allein die $\tau \alpha \dot{\alpha} \xi \iota \varsigma$ und $\lambda \dot{\varepsilon} \xi \varsigma \varsigma$. Aehnlich werden 53 die historischen $\pi$ pooi $\mu \iota \alpha$ mit den rhetorischen parallelisirt.

Auch c. 15 derselben Schrift lernen wir einen verkannten rhetorischen Terminus kennen, diesmal aber einen ganz neuen.

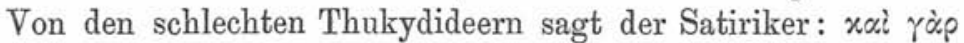

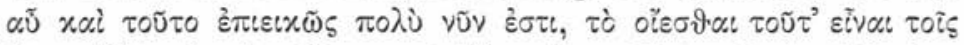

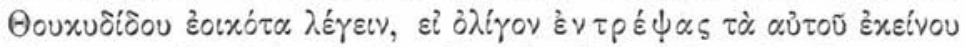

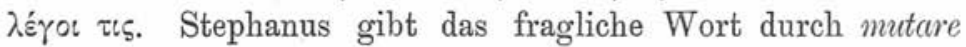

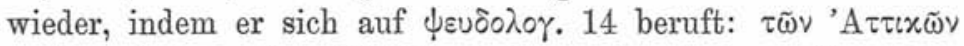

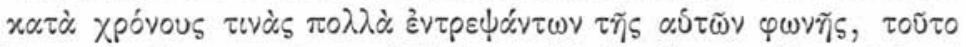

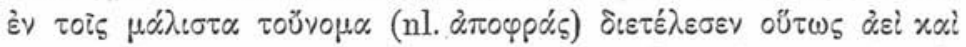

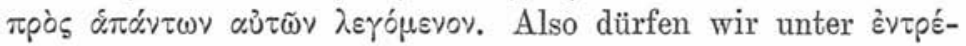
$\pi \varepsilon \downarrow v$ eine rhetorische Uebung verstehn, bei der die Originalworte vom Schüler durch andere ersetzt werden müssen etwa wie es die Scholiasten in ihren Paraphrasen machen. Das wird zur Evidenz gebracht durch die buchstäbliche Uebersetzung des Terminus bei Tacitus ann. 15, 63: et novissimo quoque momento suppeditante eloquentia (Seneca) advocatis scriptoribus pleraque tradidit, quae in vulgus edita ejus verbis invertere supersedeo.

$$
* * *
$$

Einer kleinen textkritischen Nachhilfe bedarf, wie mir

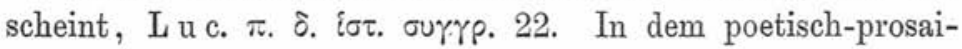




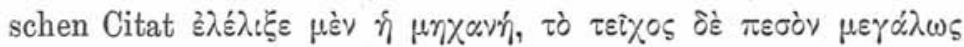
Ł̇oú $\pi \eta \sigma \varepsilon$ ist das erste Wort nur in der Bedeutung erschüttern zu brauchen; wir haben eine Situation, wie sie Livius 21, 8, 5 schildert: jam feriebantur arietibus muri ... tres deinceps turres quantumque inter eas muri erat cum fragore ingenti prociderant - einen locus communis in Belagerungsbildern. Dann ist aber ein Object bei $\varepsilon^{2} \lambda_{\varepsilon} \lambda_{\imath} \xi \xi \varepsilon$ notwendig - eben dasjenige,

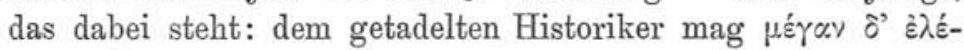

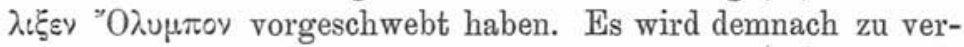

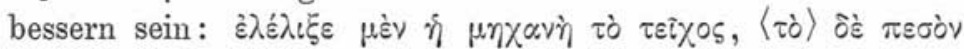

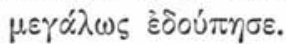

Unverständlich ist in ${ }^{*}{ }^{*}$ er Form, wie sie unsere Ausgaben bieten, folgende Stelle aus der Didoepistel Ovids: Pro meritis et siqua tibi debebimus ultra, Pro spe conjugii tempora parva peto, Dum freta mitescunt et amor, dum tempore et usu Fortiter edisco tristia posse pati (177 sqq.). Was soll hier debebimus? Dido spricht von dem, was sie bei Aeneas 'gut hat', von ihrem Haben, nicht von ihrem Soll; das alles will sie um eine kurze Frist hingeben. Dazu gehören 1) ihre Verdienste um ihn, 2) eben unser Rätsel und 3) sein Eheversprechen. Was unter 2) zu erwarten ist, lehrt V. 89 sqq.: Fluctibus ejectum tuta statione recepi, Vixque bene audito nomine regna dedi (das sind die merita, denn nun fährt sie fort): His tamen officiis utinam contenta fuissem, Et mithi concubitus fama sepulta foret (letzteres verdorben: man möchte $\mathrm{Ei}$ mihi! concubitus fama sepulta viget vermuten). Und eben das ist auch an unserer Stelle überliefert, denn die erste Hand des einzig massgebenden $\mathrm{G}$ hat debobimus, d. i. devovimus. Der Sinn ist: 'Für meine Verdienste um dich ... und was ich dir sonst noch hingeopfert habe' u. s. w.

$$
* * *
$$

Bannformeln. Mit vollem Recht sieht A. Sonny (in der russ. "philol. Rundsch." IV 1, 190) den ersten Halbvers

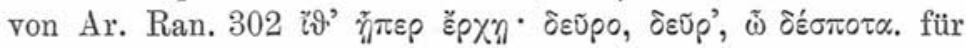
eine an die Empuse gerichtete Bannformel an; wenn er sich

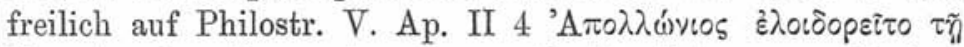
'E $\mu \pi 0^{\prime} \sigma n$ beruft, so kann man die Parallele nur mit einer 
kleinen Einschränkung gelten lassen: von den drei Beschwörungsmitteln, die das Folklore für solche Fälle anerkennt StoGgebet, Schimpf in Wort und Geberde, Bannformel - hat Apollonius das zweite, Xanthias das dritte angewendet. Aber auf Lysistr. $833 \mathrm{f}$. wirft unsere Stelle, richtig interpretirt, ein helles Licht: wenn die Heldin, wo sie den Kinesias in der bekannten Verfassung auftreten sieht, ihn mit den Worten be-

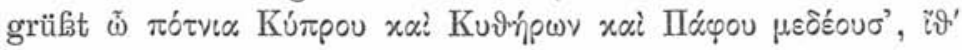

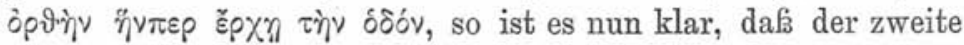

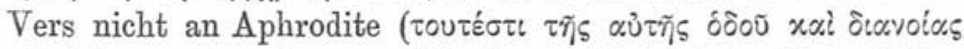
schol.), sondern eben an Kinesias gerichtet ist. Sie will ihn eben durch die Bannformel als ein Ungeheuer kennzeichnen

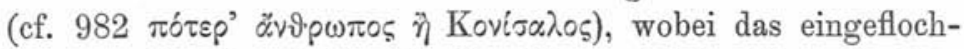
tene zweideutige $\partial \rho \vartheta \eta^{\prime} \nu$ die Parodie markirt. Eine ähnliche spassige Bannformel in ähnlicher Lage lesen wir bei Boccaccio Dec. VII 1: Fantasima, fantasima che di notte vai, a coda ritta ci venisti, a coda ritta te n' andrai.

Eine Bannformel glaube ich auch bei Petron. $64 \mathrm{zu}$ erkennen: osculatique mensam rogamus Nocturnas, ut suis se teneant, dum redimus a cena. Gewöhnlich werden die Worte des Gebets für unvollständig gehalten. Wehl ergänzt triviis, Bücheler und Friedländer sedibus. Im Sinne mag man dieses oder ähnliches ergänzen; daß3 der Text als solcher vollständig ist, dafür scheint mir eine bislang mißsverstandene Horazstelle zu sprechen. Nachdem der Dichter den lästigen Sittenprediger durch sein energisches jam desine vergeblich hat zur Ruhe bringen wollen, sucht er (sat. II 3, 324) seinem Redestrom Einhalt zu tun, indem er ihm teneas, Damasippe, tuis te zuruft. Die Aehnlichkeit mit der petronianischen Bannformel ist unverkennbar; und wie Horaz den Petronius vor textkritischen Eingriffen schützt, so gibt andrerseits Petronius für Horaz die richtige, humorvolle Interpretation weich' von mir, Unhold! an die Hand.

$$
\text { * * * }
$$

Ovid fast. I 123 läßt sich sanguine letifero kaum halten, wie am besten der Rettungsversuch H. Peters weil es vergossen den Tod bringt beweist; die Verbesserung luctifero drängt sich 
von selber auf. Ebenda ist nach 180 eine Lücke anzunehmen, die den Uebergang vor den sonstigen principia zum Jahresanfang enthielt. Weiter 286 ist niveo cado sachlich anstößig; das paläographisch gleichwertige luteo wird auch durch Mart. IV 46, 16 Hispanae huteum rotae toreuma nahegelegt. - Der Anfang des schönen Feraliengedichts (II 533) lautete früher nach allerdings geringeren Handschriften kraft- und weihevoll also: Est honor et tumulis; animas placate paternas Parvaque in extinctas munera ferta pyras! jetzt hat man mit dem schwächlichen placare und ferre sowie mit dem sinnlosen extructas den Anschluf an die 'bessere Ueberlieferung' (als ob bei solchen palaeographischen Aequivalenten von einer Ueberlieferung auch nur die Rede sein könnte!) erkauft. Es ist also auch für die Gräber eine Ehre, die Seelen der Väter zu versöhnen - denn so will es die Grammatik. Soll ferner mit (540) media testa relicta via die mit den Liebesgaben mitten auf der (appischen) Straße zurückgelassene Schale gemeint sein, so verstehe ich nicht, wie sie dem Schicksal entgieng, von Rossen, Maultieren etc. zerstampft zu werden - ganz abgesehn davon, dafs man dann nicht wufte, ob sie zum rechten oder zum linken Grab gehörte. Aber das parva petunt manes wird passend dadurch illustrirt, dafs ihnen auch eine mitten auf der Strasse aufgelesene Scherbe als Schüssel genügte - wofern man sich entschliefit, das sinnlose relicta durch sein Aequivalent relata (cf. 532) zu ersetzen (cf. Mart. VI 33 testa vetus, media sed modo fracta via). - Weiter unten wird adde preces positis et sua verba focis illustrirt durch II 314 und Plin. h. n. 22, 6, 11 immolasse ad tibicinam foculo posito, es ist somit foci keineswegs = pyra 534 und busta 551, wobei positis sinnwidrig wäre. - Der Sinn von III 419 Caesaris innumeris, quos maluit ille mereri, accessit titulis pontificalis honor wird durch Cic. Sest. $6 \mathrm{tr} . \mathrm{pl}$. . . f factus . . reTiquis honoribus non tam uti voluit quam dignus videri erklärt: jene hat er höflich abgelehnt, diese angenommen. - Wenn es 453 von Pegasus heift huic supra nubes et subter sidera lapso, so ist es eine Märchenreminiscenz; der Ausdruck kehrt in russischen Märchen und Sagen wieder (z. B. von Ilja Muromec: „er sprengt oberhalb des stehenden Baumes, kaum unterhalb der eilenden Wolke"). - Die Eigentümlichkeit der lex Car- 
seolana, deren aitıov IV 679 f. erzählt wird, bestand nicht etwa darin, daf man den gefangenen Fuchs nicht am Leben lief (das geschieht wohl nirgends), sondern darin, dafs er nicht mit dem Eisen getödtet, sondern verbrannt wurde; es scheint demnach 709 nam vivere captam in namque icere $c$. geändert werden zu müssen.

$$
* * *
$$

Die u. a. auch aus den archäologischen Handbüchern und Schriftquellen bekannte Beschreibung des myronischen Discobolen bei Lucian Philops. 18 leidet noch immer an einem Textfehler, dessen Heilung übrigens jedem, der einmal auf ihn aufmerksam geworden ist, leicht einfallen wird. Wenn es da

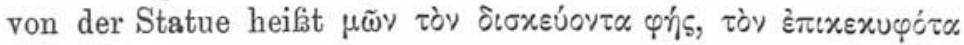

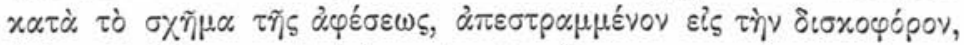

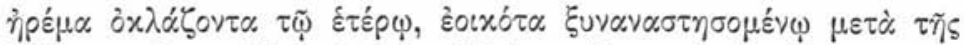

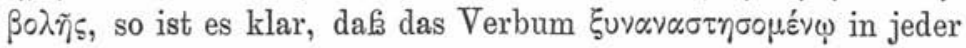
Hinsicht anstössig ist: weder kommt die Praeposition oúv zu ihrem Recht, da ja das Verbum auf den Diskus keine Anwendung findet, noch hat dieses letztere die ihm zukommende Bedeutung (der Sitzende und der Liegende $\dot{2} v^{i} \sigma \tau \alpha \tau \alpha \iota$, der ge-

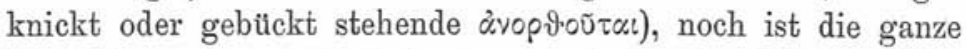
Vorstellung irgendwie bedeutsam und charakteristisch. Wer das epigrammatische der hellenistischen und sophistischen Statuenekphrasen, ihre Vorliebe für die ḋóv $\alpha \tau \alpha$ ( $\tau \dot{\alpha} \chi \alpha, x \eta p \dot{\text {, }}$

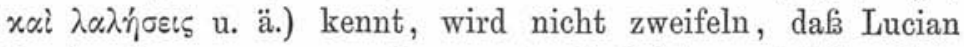

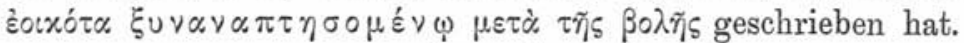

$$
\text { * * * }
$$

Die Reste rhetorischer Gelehrsamkeit, die uns als $\Delta \eta \mu \eta$ -

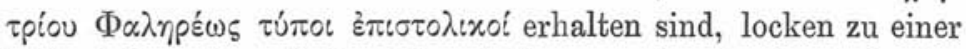
historisch-systematischen Verarbeitung, wie sie dereinst eine dringend erwünschte "Geschichte des antiken Briefstils" wird geben können; es steckt trotz des etwas öden Schematismus mancher feine Gedanke drin. Hier nur ein paar kritisch-exe-

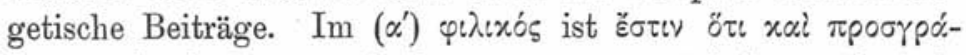

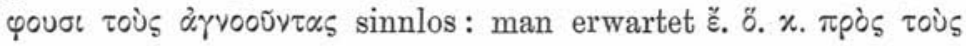

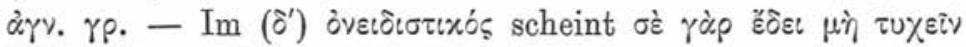

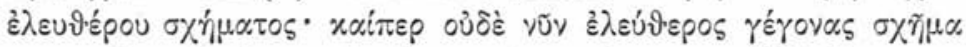

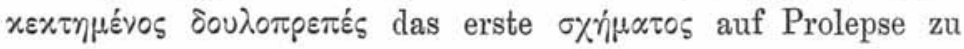




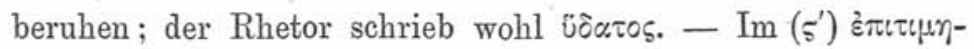

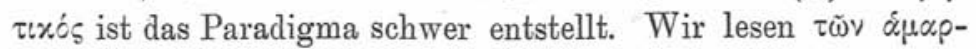

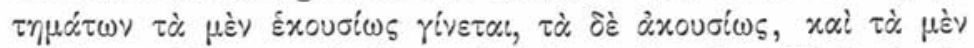

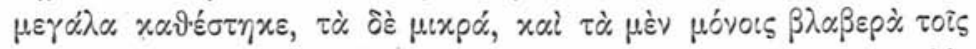

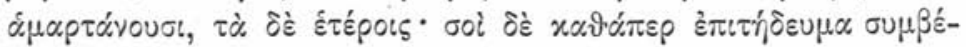

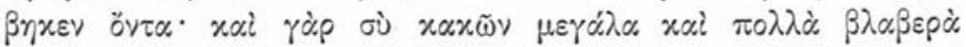
oı $\alpha \pi \varepsilon ́ \pi \rho \alpha \xi \alpha$. Hier möchte ich im zweiten Satze zweifelnd

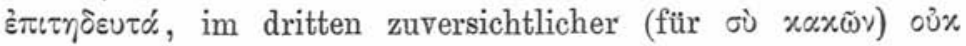

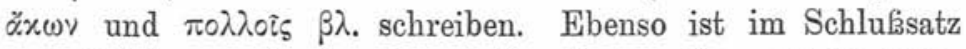

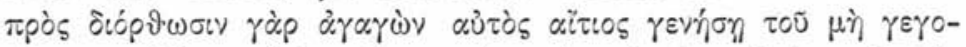

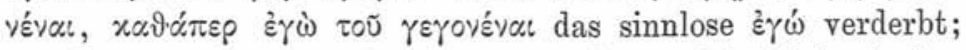

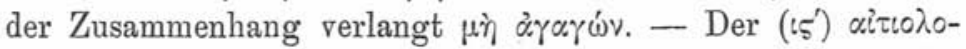

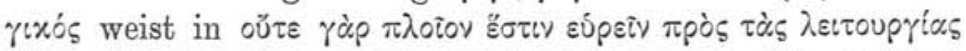

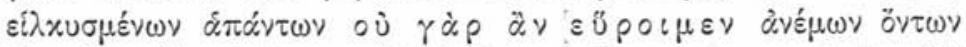

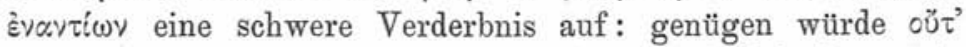

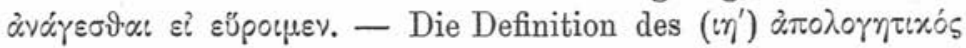

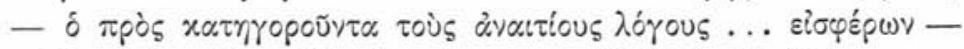

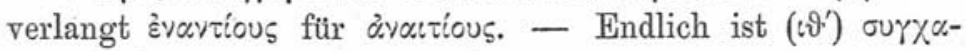

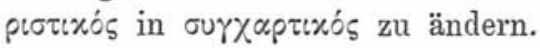

$$
* * *
$$

Ich hatte Philol. 50, $139^{7}$ in den Metamorphosen Ovids eine Dittographie nachgewiesen, dadurch entstanden, daf zwei Fassungen derselben Stelle im Archetypus zu einem Ganzen verschmolzen worden sind; ähnlich liegt die Sache I 545 (Daphne). Hier hat der Marcianus victa labore fugae tellus ait hisce, vel istam qua nimium placui mutando perde figuram, wobei jedoch der erste Halbvers i. r. also lautet: spectans peneidas undas. Vollzogen ist die Verschmelzung in den beiden geringeren Handschriften, mit deren Hilfe wir leicht folgende zwei Fassungen herausschälen: 1) Victa labore fugae: Tellus, ait, hisce vel illam, Qua nimium placui, mutando perde figuram!, und 2) Victa labore fugae, spectans Peneidas undas: Fer pater, inquit, opem, si flumina numen habetis! Quae facit ut laedar, mutando perde figuram. Daf3 beide Fassungen ovidianisch sind, ist nicht $\mathrm{zu}$ bezweifeln ; insbesondere beruht die erste auf guter Tradition, die wir aufer den von Sybel myth. Lex. citirten

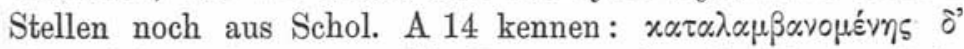

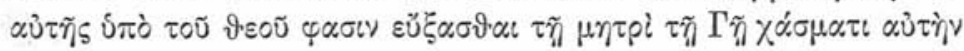




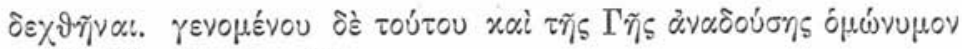

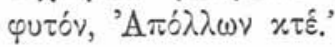

$$
\text { * * * }
$$

Eine Corruptel eigener und wohl seltener Art lernen wir Ov. met. V 478 aus dem Marcianus kennen. Hier heißt es von Ceres sinnlos: paritique aetate colonos Ruricolasque boves leto dedit, und erst die geringeren Handschriften bieten das richtige parilique irata colonos etc. Der Schreiber hat sich durch VIII 631 pia Baucis anus paritique aetate Phitemon beeinflussen lassen.

$$
* * *
$$

Die Rückkehr des Frühlings wird Ov. trist. III 12 beschrieben: dort heifst es u. a. (8) ... indocitique loquax gutture vernat avis; utque malae crimen matris deponat hirundo sub trabibus cunas tectaque parva facit. Nun ist es klar, dab unter dem Vogel V. 8 eben die Schwalbe gemeint ist (cf. fasti I 158; Meineke zu Theokr. p. 439 f.; Nauck MGR II 362); also ist hirundo V. 9 für ein Glossem zu halten, das am ehesten ein Attribut zu trabibus, etwa acernis, verdrängt haben kann. Etwas unten 19 lusus equis nunc est, levibus nunc luditur armis ist mir das Epitheton zu armis unverständlich; man möchte eine Umstellung vermuten, durch die das ursprüngliche lusus nunc levibus armis alterirt worden sei. - Ex Ponto IV 14 klagt Ovid, daßs ihm die Tomiten wegen seiner Elegien zürnen: ergo ego, ne scribam, digitos incidere cunctor, Telaque adhuc demens, quae nocuere, sequor? Tela sequi ist unverständlich; und da es Hor. ep. I 8, 11 quae nocuere sequor heist, so möchte ich unter Berufung auf die soeben besprochene Corruptel met. V 478 vermuten, daß infolge einer unzeitigen Reminiscenz das ursprüngliche telaque .. quae nocuere fero zu Schaden gekommen ist.

$$
\text { * * * }
$$

Einen etwas absonderlichen Namen finden wir CIL V 6482 M. Domitius M. (man ergänzt l. oder f.) Claruscantus. Mommsen verhält sich dem Cognomen gegenüber skeptisch - in der Tat ist er mehr nach den Gesetzen der indianischen als der römischen Onomatologie gebildet. Leider ist die Inschrift nach einer Copie Bruzzas edirt; eine Einsicht des Originals würde 
wahrscheinlich lehren, dafs die Standesbezeichnung in dem zweiten Buchstaben des Cognomens zu suchen ist, so daf der Mann - ein sehr häufiger Fall - zwei Patrone (M. und C.) gehabt haben würde, der Rest aber - ARVSCANTVS vielmehr als ABASCANTVS gelesen werden muk.

* * *

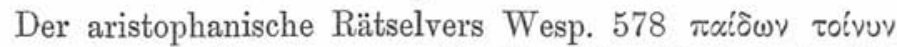

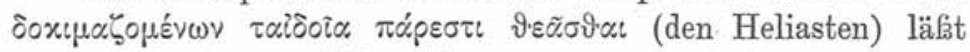
sich durch Aristot. 'A $\vartheta$. $\pi 0 \lambda$. 42, wie mir scheint, völlig befriedigend erklären. Wir lernen daraus, das beim Eintragen der Knaben in die Bürgerrollen durch die Demoten eine dop-

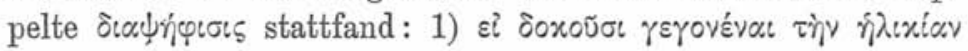

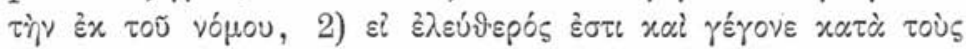
vópous. Das Urteil der Demoten war jedoch nicht endgiltig, seine Cassation ist a priori auf doppeltem Wege denkbar, auf dem der Revision von oben und auf dem der Appellation von unten - wobei es in der Natur der Sache begründet ist, daf der eine Weg im Falle unrechtmäßiger Eintragung, der zweite im Falle unrechtmäfiger Ablehnung beschritten wurde. Revisionsinstanz war natürlich die Bule, von der auch weiter die Rede ist - freilich nur dem Resultat der ersten ócoழ̣

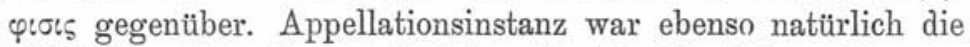
Heliaia, und auch von ihr ist weiterhin die Rede, aber nur

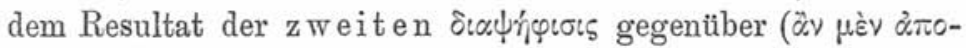

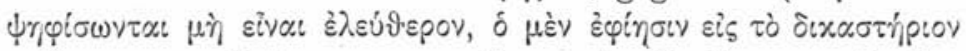

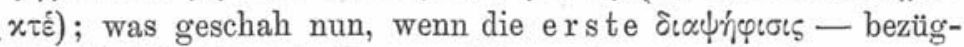
lich der Volljährigkeit - zu Ungunsten des Knaben ausgefallen war? Dafs bei Aristoteles auch von ihr die Rede war,

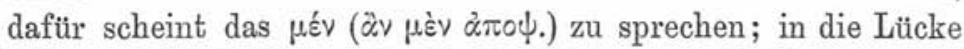
tritt nun Aristophanes ein. In der Tat waren alle Bedingungen für eine в̌ Appellation zu unterwerfen, die erste aber nicht.

Bei dem hochconservativen Standpunct, den der neueste Herausgeber und Erklärer des Properz der Ueberlieferung gegenüber einnimmt, möchte man es von vornherein für unwahrscheinlich halten, daßs auch nur an einer Stelle die handschriftliche Lesart gegen ihn in Schutz genommen werden 
müßate. Und doch glaube ich nachweisen zu können, daß3 er Prop. IV 3, 48 ohne Not von der Ueberlieferung abgewichen ist. Arethusa schreibt:

Nec me tardarent Scythiae juga, cum pater altas

Africus in glaciem frigore nectit aquas.

Hier nimmt Rothstein an Africus Anstoß, an dessen Stelle er (mit Eldick) astricto setzt; und da auch die übrigen Herausgeber ändern, wird es nützlich sein, die Stelle heranzuziehen, welche die unsrige in ihrer handschriftlichen Fassung schützt und erklärt. Es ist Liv. 21, 58, 8 (Hannibals Appenninenübergang): et mox aqua levata vento cum super getida montium juga concreta esset, tantum nivosae grandinis dejecit, ut omnibus omissis procumberent homines etc. Für das generelle vento hat der Dichter das specielle Africus pater geschrieben, im übrigen ist die Uebereinstimmung so schlagend (juga; aqua; levata $\sim$ altas; concreta $\sim$ nectit; getida $\sim$ frigore), daf man vermuten könnte, der Dichter hätte den Historiker vor Augen gehabt - wozu die Zeit recht gut passen würde.

$$
* * *
$$

Oefter wird man mit dem trefflichen Herausgeber, wie soeben angedeutet, in entgegengesetztem Sinne rechten müssen - ohne deswegen den Dank zu vergessen, der seiner hervorragenden Leistung gebührt. Wie schön schlof IV 9 nach Bährens' Umstellung mit dem feierlichen Gebete ab! Nun hat 73 hunc seine Beziehung verloren, und das ganze Distichon hängt als trockene Fußnote zu 72 Sancte über. Wie abscheulich ist, wenn man sich nur einigermassen hineindenkt, IV 3, 56 illa (das Hündchen) tui partem vindicat una toro - und wie alt ist die sichere Verbesserung tori! Am stärksten muf man aber bei IV 5, 15 aufseufzen, wo man nun von der Hexe die zusammenhanglosen, wie gestammelten Verse liest:

posset et intentos astu caecare maritos, cornicum immeritas eruit ungue genas, während schon Vahlen durch die glänzende Emendation posset ut Licht und Ordnung geschaffen hat. Ich kenne Vahlens Gründe nicht, glaube sie aber erraten zu können. Die Hexe blendet die Krähen, um dadurch die Männer für die Schleichwege ihrer Frauen blind zu machen: das ist ein Musterbeispiel 
eines weitverbreiteten Sympathiezaubers. Sehr nahe der Properzstelle kommt die Hexe bei Ovid fasti II 577, die zu ähnlichen Zwecken den Mund eines Fischchens versiegelt und vernäht: hostiles linguas inimicaque vinximus ora, erklärt sie selber.

$$
\text { * } * *
$$

Ein Hauptrorzug des neuen Commentars liegt unstreitig in der Sorgfalt, womit der Erklärer der in die einzelnen Wörter hineingebannten Inhaltsfülle nachgegangen ist, mag nun diese in einer concreten Vorstellung liegen, oder aber in einem eigenartigen Stimmungszauber, der unsern Dichter, diesen echten Sprossen der hellenistischen Decadenz, vor allen anderen auszeichnet. Properz ist der Dichter der Emphase schlechthin das Wort im antiken, nicht im modern confusen Sinne ver-

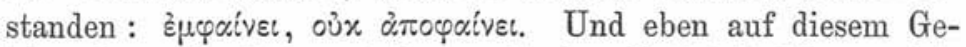
biete kann die Interpretation auch über Rothstein hinaus vielfach gefördert werden. Wenn Vertumnus IV 2, 13 sagt:

prima mihi variat liventibus uva racemis

et coma lactenti spicea fruge tumet,

so ist ja das Halbreife der Traube recht anschaulich durch liventibus angedeutet. Das gleiche ist aber auch für lactenti anzunehmen: wer je ein halbreifes Korn der Brotfrucht zerdrückt hat, weiß, daß es nichts festes, sondern eine milchweiße Flüssigkeit enthält. - Anders als der Herausgeber würde ich auch I 20, 41 fassen, eins der entzückendsten Disticha, die dem Dichter je gelungen sind: Hylas

... modo formosis incumbens nescius undis

errorem blandis tardat imaginibus.

Wenn er den Krug versenkte, würde er die liebliche Täuschung zerstören; deswegen tut er es eben noch nicht. Daher tardat.

$$
* * *
$$

Stimmungszauber ist es auch, wenn der Dichter einen schönen Moment dadurch zum Verweilen nötigt, daß er seine Beschreibung mit leicht variirten Ausdrücken noch einmal gibt; ein Musterstück dieses in seiner Wirkung fast musikalischen Kunstgriffs gibt uns Kallimachos selber in seiner einzigen uns erhaltenen Elegie (Hymn. 5, 70 f.) 


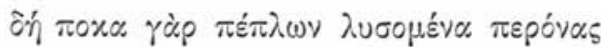

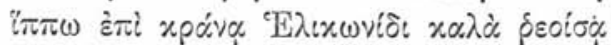

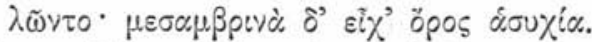

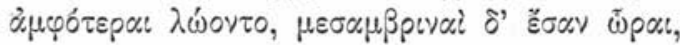

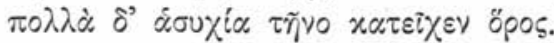

Seine Stilechtheit bezeugt uns der griechische Roman, der ja mit tausend Fäden an die hellenistische Elegie geknüpft ist - und zwar in demjenigen seiner Vertreter, welcher der alexandrinischen Idylle am nächsten steht, Longus; man ver-

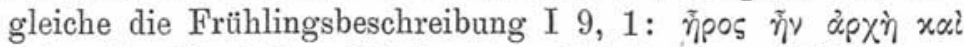

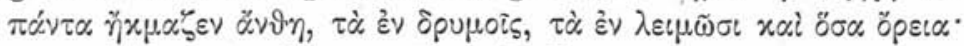

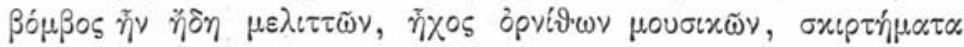

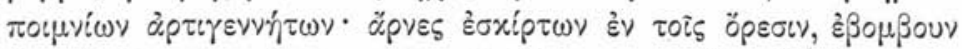

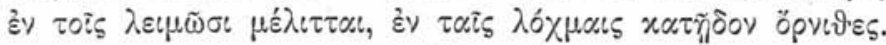

$$
* * *
$$

Mit dieser Wiederholungsfigur ist nicht zu verwechseln eine andere, viel gewöhnlichere, die durch eine Parenthese bedingt ist; es ist ein Wiederaufnehmen des Gedankens, das man sich durch "also, wie gesagt ..." eingeleitet denken kann. Von dieser Art ist bei demselben Kallimachus V $13 \mathrm{ff}$.

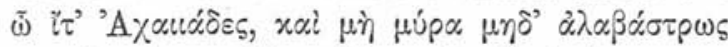

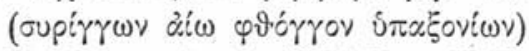

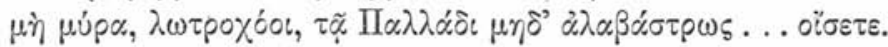

Und auch ein zweites Beispiel ist aus derselben Elegie anzuführen, $60 \mathrm{ff}$.

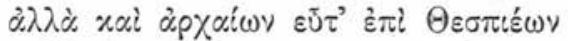

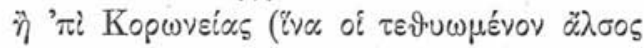

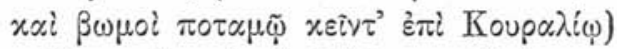

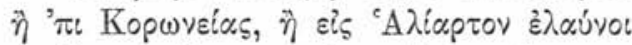

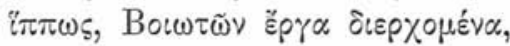

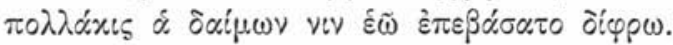

Hiebei habe ich, um die nötige Parenthese zu erzielen,

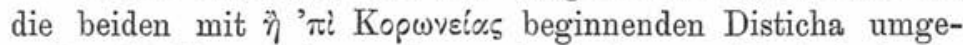
stellt; die Herausgeber nehmen eine schwerere Verderbnis des Textes an, wozu mir keine Nötigung vorzuliegen scheint.

Wie eng sich die gelehrte alexandrinische Elegie an die Tragödie anschließen konnte, dafür bietet ebendasselbe Gedicht 
des Kallimachos ein auffallendes, m. W. noch nicht beachtetes Beispiel, V. 18 ff.:

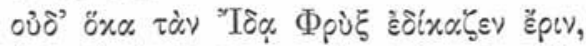

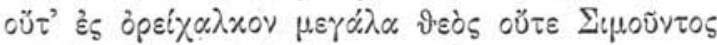

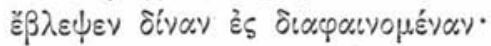

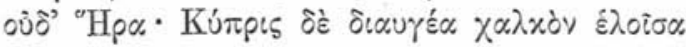

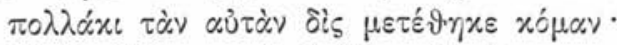

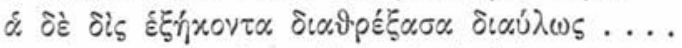

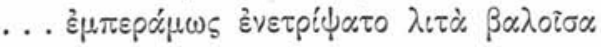

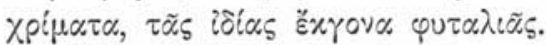

Man könnte geneigt sein, hier ein Musterstück jener wohlbekannten alexandrinischen Kleinmalerei zu sehn; doch das wäre eine Täuschung. Das ganze Bild mit allen seinen Einzelheiten stammt aus dem sophokleischen Satyrspiel Kpiors, aus dem uns Athenaeus ( $687 \mathrm{c}=$ fr. $334 \mathrm{~N}$ ) das uns interessirende

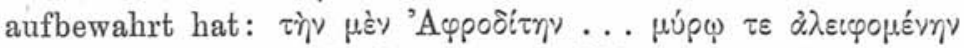

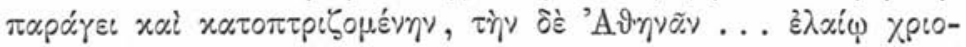

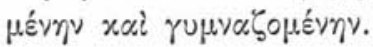

Um so weniger Anstof wird es erregen, wenn ich auch beim römischen Kallimachos eine ähnliche Anlehnung an eine sophokleische Tragödie nachweise. Properz III 6 geht auf die 'Trachinierinnen' zurück. Die sämmtlichen Hauptpersonen des Dramas - Herakles, Deianira, Iole, Lichas - finden wir mit veränderten Namen, oder auch ohne solche, in den bürgerlichen Verhältnissen der Elegie wieder. Zunächst ist die Situation dieselbe, wie nach V. $632 \mathrm{der}$ Tragödie. Es ist der Geliebten des Dichters hinterbracht worden, daf er es mit einer anderen halte; der Bote, hier Lygdamus genannt, teilt ihm nun mit, in welcher Verfassung er seine Herrin verlassen habe. Die Warnung, die dem Lichas in der Tragödie von

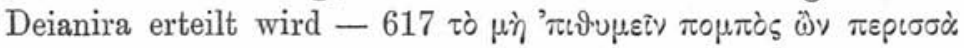
бра̃ - muli Lygdamus hier vom Dichter selber hören, 5 omnis enim debet sine vano nuntius esse; seine Erzählung reproducirt recht getreu die einzelnen Momente der Leidensgeschichte

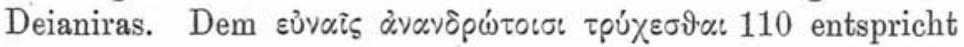
wörtlich 23 vacuo solam tabescere lecto, und wie das Weib des

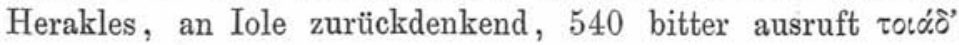




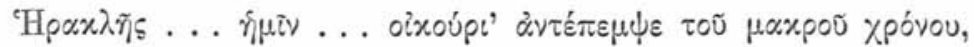
so hören wir auch hier aus dem Munde der Verlassenen die vorwurfsvolle Frage 19 haec te teste mihi promissa est, Lygdame, merces, wobei unter merces eben die Nebenbuhlerin verstanden wird. Wenn nun aber gar die Geliebte, ohne von der Nebenbuhlerin etwas zu wissen, in die entschiedene Klage ausbricht 25 non me moribus illa, sed herbis improba vicit - was im folgenden näher ausgeführt wird, - so entspricht dieser Sachverhalt durchaus meiner Interpretation gewisser Stellen aus den 'Trachinierinnen', worüber ich meine 'Excurse' zu dieser Tragödie 55, 530 ff. dieser Zeitschrift nachzulesen bitté.

$$
* * *
$$

Diese letzteren kann nun jeder nach Belieben kritisiren; nur soll er sich nicht die Kritik durch falsche Referate erleichtern. Wenn es in Bursians Jahresbericht für 1898 I 139 von ihnen u. a. heift: "Heralles Liebe zu Iole ist sein erster und einsiger Treubruch". Da V. 544 das Gegenteil mit aller Bestimmtheit sagt, wird dieser als unecht erliärt. Reine Willkiir! - so schwebt dieser Ausruf in der Luft: der Berichterstatter hat vielleicht die Güte, S. 517 nachzulesen, - wo über V. 544 "ohne Praejudiz" gehandelt und die Athetese aus ganz unabhängigen Gründen (hauptsächlich wegen des Widerspruchs mit 541) ansgesprochen wird - und sich fortan auch

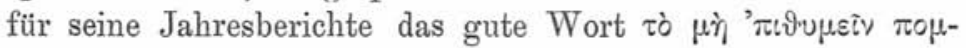

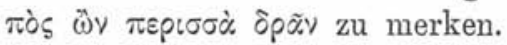

Th. Zielinsti. 